\title{
Akute obere gastrointestinale Blutung
}

Robert Baumbach, Siegbert Faiss, Wolfgang Cordruwisch, Carsten Schrader

Bei gastrointestinalen Blutungen ist schnelles Handeln gefragt - in 10\% der Fälle enden sie tödlich. Sind die Patienten instabil und der Blutverlust groß, muss notfallmäßig endoskopiert werden. Unabhängig von der Blutungsursache ist die dringlichste Maßnahme immer die Stabilisierung des Kreislaufs. Erst dann wird eine passende Therapiestrategie ausgewählt: Die Möglichkeiten reichen von Protonenpumpeninhibitoren über Injektionstherapie und Fibrinkleber bis hin zur Varizenligatur oder gar der Notfall-Laparotomie als

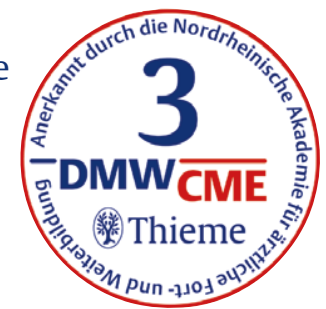
Ultima Ratio.

\section{Der konkrete Fall}

Anamnese I Eine 57-jährige Patientin mit degenerativem Rückenleiden und chronischem NSARAbusus berichtet über Hämatochezie und Übelkeit ohne Erbrechen oder Bauchschmerzen seit dem Vortag. Blutverdünnende Medikamente nimmt sie nicht ein. Magen- oder Duodenalulzera hat sie bisher nicht. Im Verlauf bemerkt sie eine progrediente Schwäche und kollabiert, bevor sie in die zentrale Notaufnahme kommen konnte.

Körperliche Untersuchung I Die Untersuchung ergibt folgende Befunde:

- wach und orientiert

- blasses Hautkolorit

- Blutdruck 85/55 mmHg

- Puls rhythmisch, $125 / \mathrm{min}$

- Herztöne rein

- Lunge perkutorisch und auskultatorisch unauffällig

- Darmgeräusche regelrecht

- Bauchdecke weich

- kein Druckschmerz

- rektodigital frisches und koaguliertes Blut am Fingerling

- keine Resistenz palpabel

Diagnose I Im Labor fällt eine normochrome Anämie mit einem Hämoglobinwert $(\mathrm{Hb})$ von $6,4 \mathrm{~g} / \mathrm{dl}$ auf. Initial erhält die Patientin 11 RingerAspartat und 2 Erythrozytenkonzentrate (EK). Die Ösophagogastroduodenoskopie am selben Tag ergibt ein ca. $13 \mathrm{~mm}$ durchmessendes, nicht stenosierendes Ulcus duodeni in der Bulbusspitze vorderwandig-minorseitig mit peripherem Gefäßstumpf ohne aktive Blutung. Es handelt sich am ehesten um eine NSAR-induzierte Ulcusduodeni-Blutung, Forrest IIa.

Therapie | Es wird zunächst eine Injektionstherapie am Ulkusrand mit insgesamt $4 \mathrm{ml}$ Suprarenin vorgenommen. Im Anschluss werden 2 Metall- clips ohne Blutungsreaktivierung appliziert. Ein abschließender Helicobacter-pylori-Schnelltest ergibt einen negativen Befund.

Verlauf I Der Hb stieg nach Transfusion adäquat um 2 Punkte an und blieb im weiteren Verlauf unter Protonenpumpeninhibitor(PPI)-Therapie stabil. Am 3. Tag wurde die Patientin unter oraler PPITherapie nach Hause entlassen.

Anamnese und klinischer Befund aussagekräftig I Gastrointestinale Blutungen kommen täglich in der Notaufnahme eines jeden Krankenhauses vor. Die Anamnese und der klinische Befund lassen differenzialdiagnostisch in erster Linie auf eine obere gastrointestinale Blutung mit hoher Blutungsintensität - z.B. bei Ulcus duodeni - oder auf eine untere GI-Blutung (z.B. Angiodysplasieoder Kolondivertikelblutung) schließen. Der Zusammenhang von Anamnese und klinischem Befund lässt häufig

- die Blutungslokalisation,

- die Blutungsintensität und

- die Diagnose

abschätzen. So lässt sich die Indikation für die Dringlichkeit und Art der endoskopischen Diagnostik und Therapie stellen.

Definition | Diese Art der akuten Blutung gehört zu den häufigsten Notfallsituationen im internistischen Alltag. Gastrointestiale (GI) Blutungen werden in obere, mittlere und untere Blutungen unterteilt. Die oberen GI-Blutungen reichen bis zum Treitzschen Band, die mittleren vom Treitzschen Band bis zur Bauhinschen Klappe und die unteren sind ab der Bauhinschen Klappe lokalisiert.

Akute GI-Blutungen sind in der Mehrzahl im oberen Gastrointestinaltrakt lokalisiert und ca. in der Hälfte der Fälle durch peptische Ulzera bedingt. 


\begin{tabular}{ll}
\hline Ursache & Häufigkeit \\
\hline Ulcus duodeni & $27 \%$ \\
Ulcus ventrikuli & $24 \%$ \\
Ösophagusvarizen & $19 \%$ \\
Erosionen & $13 \%$ \\
Refluxösophagitis & $10 \%$ \\
Mallory-Weiss-Läsionen & $7 \%$ \\
Tumor & $3 \%$ \\
Angiodysplasie & $1 \%$ \\
nicht identifiziert & $6 \%$
\end{tabular}

Tab. 1: Ursachen oberer Gl-Blutungen in der Reihenfolge der Häufigkeit [2].

Epidemiologie I Den größten Anteil der GI-Blutungen machen die oberen Blutungen mit 80$85 \%$ aus. Die Hälfte davon entsteht durch peptische Ulzera. Eine untere GI-Blutung tritt in 10-15\% der Fälle auf. Die mittlere GI-Blutung bleibt mit 3-5\% ein seltenes Ereignis.

$75 \%$ aller Blutungen sistieren spontan, $20 \%$ sistieren spontan und rezidivieren, und nur $5 \%$ sind massiv und sistieren nicht.

Die Schwierigkeit besteht darin, diese $5 \%$ als solche frühzeitig zu erkennen und zeitnah zu diagnostizieren und therapieren.

Inzidenz | Aktuelle Daten zur oberen GI-Blutung zeigen eine Inzidenz von 86-160/100000 und eine Mortalität von ca. 10\% [1]. > Tab. 1 führt die Ursachen einer oberen GI-Blutung in der Reihenfolge ihrer Häufigkeit auf [2].

\section{Anamnese}

Leitsymptome | Anhand der Leitsymptome und der Blutungsintensität versucht die allgemeine Anamnese die Lokalisation der Blutung abzuschätzen. Die Leitsymptome einer GI-Blutung sind

- Hämatemesis (Bluterbrechen),

- Hämatinerbrechen,

- Hämatochezie und

- Meläna (Teerstuhl).

Hämatemesis und Hämatinerbrechen I Diese sind in den allermeisten Fällen Zeichen einer oberen GI-Blutung. Hämatin entsteht durch Kontakt mit Magensäure und signalisiert eine weniger intensive Blutung als Hämatemesis. Bei Hämatemesis wird das Blutvolumen erbrochen, bevor sich Hämatin bilden kann.

Meläna | Teerstuhl ist ebenfalls meistens Zeichen einer oberen GI-Blutung, kann selten aber auch durch eine mittlere GI-Blutung (z.B. Dünndarmdivertikelblutung) oder bei langsamer Passage auch Ausdruck einer unteren GI-Blutung sein (z.B. rechtseitige Kolon-Divertikelblutung). Es ensteht durch langsame Passage von wenigstens 50-200 ml Blut und bakteriellen Abbau im Kolon. Einerseits können zwischen Blutung und Auftreten von Meläna einige Stunden vergehen, andererseits kann Meläna nach Sistieren einer Blutung noch Tage nachweisbar sein.

Hämatochezie | Bei unterer GI-Blutung wird meist frisches Blut im Stuhl beobachtet. Eine massive mittlere oder obere GI-Blutung kann auch ohne Erbrechen - wie bei der oben beschriebenen Patientin - mit dem Leitsymptom Hämatochezie einhergehen. Bei Hämatochezie ist wie bei Hämatemesis die Blutungsintensität so hoch bzw. die Lokalisation der Blutung so weit distal, dass die Passage im Kolon zu schnell ist, als dass Meläna entstehen kann.

Die Übergänge von Hämatinerbrechen und Hämatemesis sowie Meläna und Hämatochezie sind jedoch fließend.

Art der Blutung | Die Blutungsintensität entspricht dem Blutungsvolumen pro Zeit. Dieses wird abgebildet durch MCV, Hb und Hämodynamik. Bei chronischen Blutungen zeigt sich durch den Eisenverlust vor allem ein erniedrigtes MCV und $\mathrm{MCH}$, ggf. mit erniedrigtem $\mathrm{Hb}$ bei stabilem Kreislauf. Bei akuten nicht fulminanten Blutungen besteht eine normozytäre Anämie ohne Kreislaufdepression. Bei einer akuten fulminanten Blutung ist sowohl das MCV als auch der $\mathrm{Hb}$ anfangs noch normal - es hat noch kein relevanter Verdünnungseffekt durch einströmende Extravasalflüssigkeit nach intravasal eingesetzt. Diese Patienten zeigen in erster Linie einen Volumenmangelschock.

Peptische Ulzera I Bei der speziellen Anamnese wird differenzialdiagnostisch nach Hinweisen für eine Blutungsursache gefragt. Peptische Ulzera deuten z.B. auf einen NSAR-Abusus hin. Risikofaktoren für eine peptische Ulkuskrankheit sind

- Rauchen,

- Alkoholabusus,

- Alter > 65 Jahre,

- kardiovaskuläre Erkrankungen,

- schwere akute Komorbidität und

- Familien- oder Ulkusanamnese.

Allerdings geht die peptische Ulkuskrankheit häufig mit atypischen Beschwerden einher oder ist asymptomatisch.

Die typischen Symptome peptischer Ulzera sind selten.

Art, Lokalisation und zeitliches Auftreten der Beschwerden lassen häufig keine Diagnose zu. 
Varizenblutung I Fulminante Hämatemesis mit hämodynamischer Instabilität lassen an eine Varizenblutung denken bei

- Alkoholkrankheit,

- chronischer Hepatitis,

- Leberzirrhose oder

- chronischer Pfortader- oder Milzvenenthrombose.

Ca. $20 \%$ aller oberen GI-Blutungen sind durch portale Hypertension bedingt. Allerdings ist nur bei $40 \%$ der Patienten mit Leberzirrhose eine Varizenblutung Ursache einer oberen GI-Blutung.

Mallory-Weiss-Syndrom I Dabei handelt es sich um einen längsförmigen Schleimhauteinriss im Bereich des gastroösophagealen Übergangs. Bei oberen GI-Blutungen handelt es sich bei ca. 10\% um Mallory-Weiss-Läsionen. Diese bluten meistens nicht bedrohlich und bedürfen ebenfalls selten einer endoskopischen Therapie. In $80-90 \%$ sistieren die Blutungen spontan und rezidivieren in $5 \%$ der Fälle. Aktive Blutungen versorgt man in erster Linie mit einer Injektionstherapie. Als lebensbedrohliche Komplikation sollte ein Boerhaave-Syndrom nicht übersehen werden. Hierbei kommt es zum Einriss der gesamten Ösophaguswand mit Perforation ins Mediastinum.

Patienten mit rezidivierendem Erbrechen und Blutbeimengungen im Verlauf, z. B. durch Alkoholexzess, Hyperemesis gravidarum oder Zytostatikatherapie haben bis zum Beweis des Gegenteils ein Mallory-Weiss-Syndrom.

Weitere Befunde I Patienten mit hochgradiger Niereninsuffizienz lassen an Angiodysplasien denken. Akute Blutungen durch Refluxösophagitis oder Erosionen treten meistens unter Antikoagulation auf. Dabei weist die Refluxösophagitis häufig atypische Beschwerden auf oder ist asymptomatisch, v.a. bei alten, komorbiden und bettlägerigen Patienten. Patienten mit kurz zuvor durchgeführter Papillotomie oder Polypektomie haben bis zum Beweis des Gegenteils eine PostPapillotomie- bzw. Post-Polypektomieblutung. Bei Patienten mit folgenden Symptomen muss man differenzialdiagnostisch an eine aortointestinale Fistel denken:

- intermittierende und massive Hämatochezie

- ggf. auch Hämatemesis

- Kreislaufdepression

- bekannte Aortenerkrankung

- ggf. Bauchschmerzen und/oder Rückschmerzen

- Fieber

\section{Endoskopie}

Ösophagogastroduodenoskopie (ÖGD) I Mit der ÖGD gelingt es, ca. 95\% aller oberen GI-Blutungen zu diagnostizieren. Gleichzeitig kann auch eine endoskopische Therapie vorgenommen werden. Vor der Endoskopie sollten initial instabile Patienten auf der Intensivstation stabilisiert werden. Eine Intubation schützt die Atemwege, auch wenn sie selbst ein Aspirationsrisiko birgt. Während einer Notfallendoskopie führt sie zu deutlich besseren Untersuchungsbedingungen [3].

Übersicht optimieren I Eine Notfallendoskopie sollte bei den meisten instabilen Patienten und Patienten mit großem Blutverlust erwogen werden. Um die Übersichtsverhältnisse zu optimieren, sollte 20-30 Minuten vor der Endoskopie der Motilinagonist Erythromycin injiziert werden (3 mg/ kgKG). Dies senkt die Häufigkeit von weiteren Endoskopien und Transfusionen $[4,5]$. Die zusätzliche Magenspülung und Magenablaufsonde bringen keinen weiteren Vorteil für die Übersichtlichkeit und sollten unterlassen werden [6]. Sollte der Magen trotzdem mit Blutkoagel angefüllt sein, verwendet man zunächst ein Endoskop mit einem $6 \mathrm{~mm}$ messendem $\mathrm{Ab}$ saugkanal - damit können auch große Koagel abgesaugt werden.

Können Blutkoagel nicht ausreichend entfernt werden, kann es sinnvoll sein, den Patienten umzulagern: z. B. in Rechtsseitenlage oder in Oberkörperhochlagerung, um eine in Linksseitenlage nicht sichtbare Blutungsquelle zu detektieren.

Patienten mit Hämatemesis, bei denen die Blutungsquelle nicht lokalisiert werden kann, sollten überwacht und nach einem halben oder ganzen Tag eine Kontrollendoskopie erhalten.

Endoskop richtet sich nach Lokalisation | Eine isolierte Hämatochezie mit Kreislaufdepression ist als obere GI-Blutung aufzufassen - die primäre Maßnahme ist eine Notfall-ÖGD. Bei weiter distal als im Duodenum descendens vermuteter Blutungsquelle (z.B. einer Angiodysplasieblu-

\begin{tabular}{|llll|}
\hline Forrest-Klassifikation & Ulkusblutung & $\begin{array}{l}\text { Rezidiv- } \\
\text { Rate }\end{array}$ & $\begin{array}{l}\text { Endoskopie- } \\
\text { Notwendigkeit }\end{array}$ \\
\hline Forrest la & $\begin{array}{l}\text { Ulkus mit spritzender } \\
\text { Blutung }\end{array}$ & $90 \%$ & ++ \\
\hline Forrest Ib & $\begin{array}{l}\text { Ulkus mit Sicker- } \\
\text { blutung }\end{array}$ & $30 \%$ & ++ \\
\hline Forrest Ila & $\begin{array}{l}\text { Ulkus mit Gefäß- } \\
\text { stumpf }\end{array}$ & $50 \%$ & ++ \\
\hline Forrest IIb & $\begin{array}{l}\text { Ulkus mit Blutkoagel } \\
\text { Ulkus mit hämatin- }\end{array}$ & $20 \%$ & + \\
Forrest Ilc & $<5 \%$ & - \\
\hline Forrest III & $\begin{array}{l}\text { belegtem Grund } \\
\text { fibrinbelegtes Ulkus }\end{array}$ & $<5 \%$ & - \\
\hline
\end{tabular}

Tab. 2 Forrest-Klassifikation der Ulkusblutung mit Rezidivblutungsraten [3]: Die Einteilung liefert ein Kriterium für Prognose und Interventionsbedarf. 


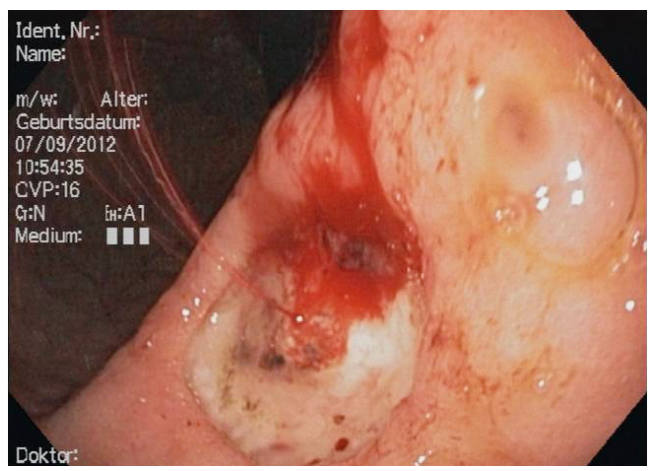

Abb. 1 Forrest-la-Ulkus mit spritzender Blutung.

tung) empfiehlt sich nach Benutzung eines normalen Gastroskops ein längeres Endoskop (z.B. Ballon-Enteroskop oder Kinderkoloskop).

\section{Angiografie}

Alternative bei unbekannter Blutungsquelle | Die Angiografie bietet eine Alternative zur Endoskopie, wenn eine Blutungsquelle nicht lokalisierbar oder endoskopisch nicht therapierbar ist (z.B. Blutung aus einem A.-lienalis-Aneurysma). Eine unklare Blutungsquelle kann in der Regel detektiert werden, wenn die Blutungsintensität mehr als $1 \mathrm{ml} / \mathrm{min}$ beträgt. Auch ohne aktive Blutung können angiografisch Blutungsquellen indirekt loksalisiert werden, indem Gefäßmalformationen oder pathologische Gefäßkonvoluten bei Tumoren erkannt werden. Mit dieser Methode ist man in der Lage, das blutende Gefäß mittels supraselektiver Katheterisierung zu embolisieren - dies ist allerdings nur in $86 \%$ der Fälle möglich [27].

\section{Klassifikation nach Forrest}

Kriterium für Intervention und Prognose I Die 1974 eingeführte Klassifikation für Ulkusblutungen nach Forrest hat sich bis in die Gegenwart als Kriterium für den Interventionsbedarf und die Prognose bewährt [7] ( Tab. 2). Bei aktiv blutenden Läsionen (Forrest-I) und bei Gefäßstumpf (Forrest-IIa) senkt die endoskopische Therapie die Rezidivblutungsrate und Mortalität. Bei Forrest-IIc- und -III-Läsionen ist eine alleinige medikamentöse Therapie angezeigt. Allerdings fällt die Einstufung gerade bei den Forrest-IIb-Ulzera je nach Untersuchung sehr variabel aus - die Klassifikation kann also nur ein grober Anhalt für die Wahrscheinlichkeit von Rezidivblutungen sein ( Abb. 1-6).

\section{Seltene Ursachen der oberen GI-Blutung}

Erosionen | Diese liegen meistens multipel vor und bluten relevant - besonders bei antikoagu-

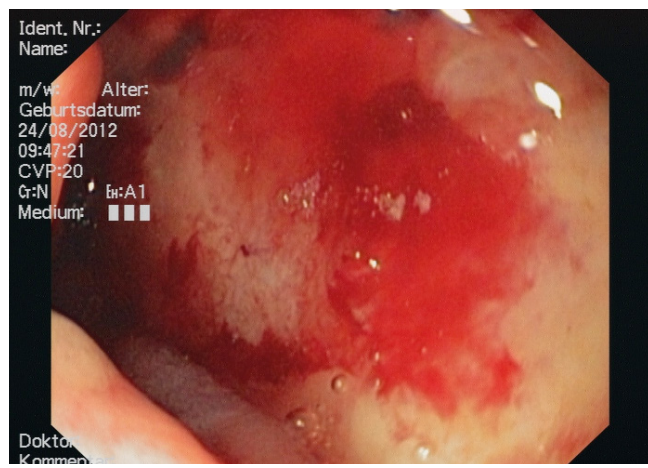

Abb. 2 Forrest-lb-Ulkus mit Sickerblutung.

lierten Patienten mit hämorrhagischer Gastritis. Liegt eine Antikoagulation vor, sollte diese z.B. mit PPSB (Prothrombinkonzentrat) antagonisiert werden. Therapie der Wahl sind PPI. Eine endoskopische Therapie ist selten nötig. Bei sehr seltenen nicht beherrschbaren Blutungen können vasoaktive Substanzen neben der Stabilisierung der Gerinnung eine sinnvolle Option sein.

Reflux | Blutungen bei Ösophagitiden liegt in den allermeisten Fällen eine primäre oder sekundäre Refluxerkrankung zugrunde (z.B. bei Magenentleerungsstörung durch diabetische Gastropathie oder Magenausgangstenose). Die Blutungen sind meistens

- multifokal,

- nicht massiv und

- treten besonders unter Antikoagulation auf. Therapie der ersten Wahl sind hier ebenfalls PPI. Einer endoskopischen Blutstillung bedarf es selten. Bei akut blutenden Refluxulzera entspricht die endoskopische Therapie der bei gastroduodenalen Ulzera.

Angiodysplasien | Gastroduodenale Angiodysplasien machen ca. $1 \%$ der oberen GI-Blutungen aus. Gehäuft kommen sie bei niereninsuffizienten Patienten vor. Therapie der Wahl ist die Argon-Plasma-Koagulation (APC).

Ulkus Dieulafoy I Ein Ulkus Dieulafoy (Exulceratio simplex) ist Ursache einer seltenen, aber potenziell lebensbedrohlichen GI-Blutung. Bezogen auf alle GI-Blutungen machen die Ulkus-Dieulafoy-Blutungen $<1 \%$ aus. Es handelt sich um einzelne aberrante arterielle Gefäße in der Submukosa bzw. Mukosa. Die Hauptlokalisation ist im proximalen Magen (v.a. Corpus minorseitig). Es gibt aber auch Beschreibungen von Manifestationen im

- Ösophagus,

- Duodenum,

- Jejunum und

- Kolon.

Wenn keine aktive Blutung vorliegt, kann die Detektion der Läsion schwierig sein: Dann ist, wenn überhaupt, wenig zu erkennen. Therapeutisch gibt es keine eindeutigen Empfehlungen. Die Me- 


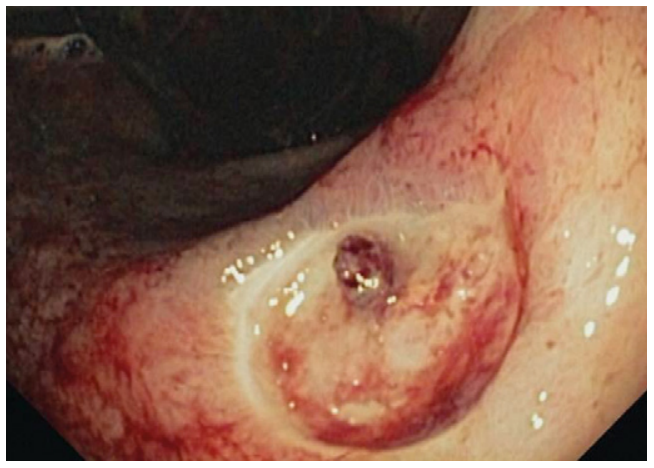

Abb. 3 Forrest-Ila-Ulkus mit Gefäßstumpf.

thoden reichen von Injektionstherapie mit Adrenalin, Histoacryl, über APC, Hämoclip bis Ligaturtherapie. Vorzugsweise ist die Injektionstherapie und Clipapplikation zu verwenden.

Aortointestinale Fisteln | Diese manifestieren sich typischerweise durch intervallartig auftretende fulminante Blutungen mit kürzer werdenden Intervallen. Amnestisch liegt eine Aortenerkrankung vor. Es wird unterschieden zwischen

- einer primären aortointestinalen Fistel mit Aortenaneurysma und

- einer sekundären aortointestinalen Fistel mit vorausgegangenem gefäßchirurgischen oder radiologischen Eingriff.

Pathogenetisch führt die konstante pulsatile Bewegung des Aneurysmas lokal zu einer progredienten Darmwanderosion. Das Intervall zwischen Intervention und Fistel kann zwischen Tagen und Jahrzehnten liegen. Aortointestinale Fisteln werden auch Aortoduodenale Fisteln genannt, weil 75\% im Duodenum, vorwiegend in der pars horizontalis duodeni, lokalisiert sind. Die Diagnosestellung ist schwierig, da die Blutungslokalisation jenseits der Erreichbarkeit einer Ösophagogastroduodenoskopie liegt. Außerdem gibt sie sich bei mangelnder Übersicht endoskopisch selten als solche zu erkennen.

Eine frühe Diagnosestellung ist aber notwendig, da diese Erkrankung unbehandelt in nahezu $100 \%$ tödlich verläuft.

Das entscheidende diagnostische Moment ist die Anamnese. Die Diagnose sichert man radiologisch mittels Angio-CT. Die Therapie besteht in einem gefäßchirurgischem Eingriff, offen oder interventionell-therapeutisch.

\section{Therapie allgemein}

Kreislaufstabilisation I Bei der akuten oberen GI-Blutung ist die Stabilisierung des Kreislaufs vordringlich. Hierzu sollten zwei großlumige und möglichst zentrale intravenöse Zugänge gelegt werden.

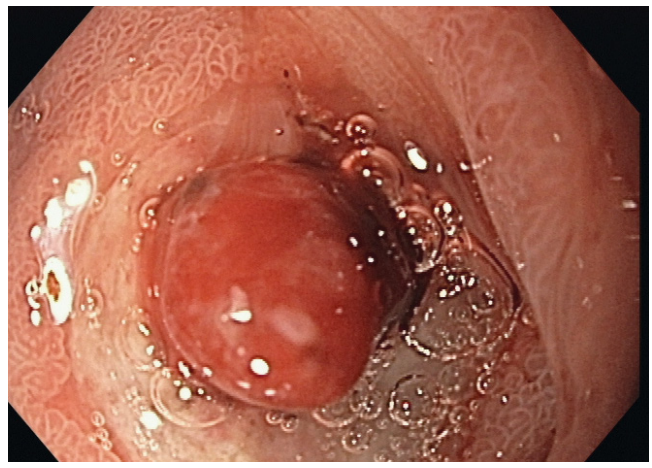

Abb. 4 Forrest-IIb-Ulkus mit Blutkoagel.

Volumengabe | Primär sollten kristalloide Lösungen gegeben werden. Bei akutem Blutverlust soll man HES-haltige kolloidale Lösungen nur dann zur Behandlung einer Hypovolämie geben, wenn die Gabe von kristalloiden Lösungen allein nicht ausreicht. Dies zeigten die Ergebnisse zweier klinischer Studien mit kritisch Kranken [8].

Transfusion von Erythrozytenkonzentraten | Zur Indikationsstellung einer Erythrozytentransfusion sollten folgende Kriterien berücksichtigt werden:

- Hb-Konzentration

- Kompensationsfähigkeit (d.h. die physiologische Fähigkeit, den verminderten $\mathrm{O}_{2}$-Gehalt des Blutes zu kompensieren)

- Risikofaktoren (z. B. KHK, Herzinsuffizienz, zerebrovaskuläre Insuffizienz) [9]

Patienten mit einem $\mathrm{Hb}$ von $<6 \mathrm{~g} / \mathrm{dl}$ sind z.B. $\mathrm{zu}$ transfundieren. Bei einem $\mathrm{Hb}$ von $<8 \mathrm{~g} / \mathrm{dl}$ besteht bei Patienten ohne eingeschränkte Kompensationsfähigkeit keine Indikation zur Transfusion. Bei eingeschränkter Kompensationsfähigkeit sollte auf den Ziel-Hb von $8 \mathrm{~g} / \mathrm{dl}$ transfundiert werden. Bei Patienten mit Zeichen der anämischen Hypoxie (z.B. Tachykardie, Hypotonie, EKG-Ischämie, Azidose) sollte der $\mathrm{Hb}$ auf $10 \mathrm{~g} / \mathrm{dl}$ angehoben werden.

Cave: Eine darüber hinaus gehende Übertransfusion ist zu vermeiden.

Im Notfall kann auch die Blutgruppe 0 ungekreuzt transfundiert werden. Pro 2 Erythrozytenkonzentrate sollte ein Plasmakonzentrat gegeben werden (z. B. Frisch Frozen Plasma / FFP).

\section{Ein Erythrozytenkonzentrat (EK) hebt den} Hb-Wert um ca. 0,75-1 g/dl an.

Monitoring und Sauerstoffgabe | Der Patient sollte auch bei fehlender Luftnot Sauerstoff über eine Nasensonde erhalten und am Monitor zur kontinuierlichen Kreislaufüberwachung liegen (Blutdruck und Pulsoxymetrie). Ist die Blutungssituation noch unklar, sollte man die Patienten auf der 


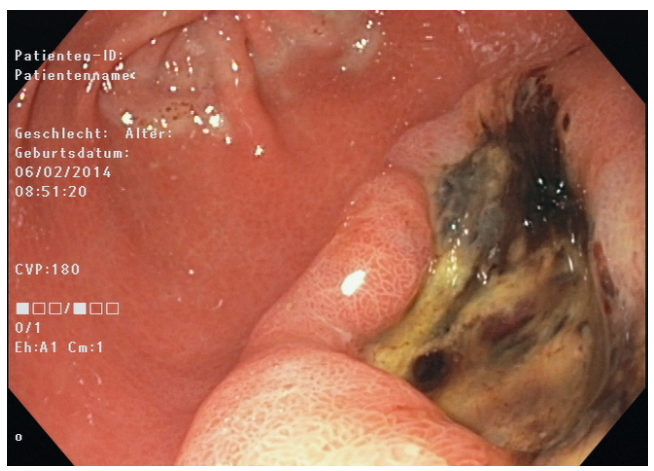

Abb. 5 Forrest-Ilc-Ulkus mit hämatinbelegtem Grund.

Intensivstation oder der Intermediate Care Station überwachen.

\section{Therapie der peptischen Ulkusblutung}

Inzidenz | Die Ulkusblutung ist die häufigste Ursache von oberen GI-Blutungen. Es stehen pharmakologische, endoskopische, angiologische und chirurgische Therapieoptionen zur Verfügung. Die Inzidenz der peptischen Ulkusblutung liegt altersübergreifend bei 22/100000. Die 30-TageMortalität liegt bei $2 \%$. Mit zunnehmendem Alter steigen sowohl Inzidenz als auch 30-TageMortalität an - bei Patienten $>80$ Jahre liegt die Mortalität bei $8 \%$ [10].

Protonenpumpeninhibitoren (PPI) | Grundlage jeder Ulkustherapie ist die Gabe von PPI. Allerdings bieten PPI als Monotherapie hinsichtlich Rezidivblutung, Operation und Letalität keinen Vorteil im Vergleich zu Placebo. Vor der Notfallendoskopie soll eine hochdosierte Gabe von PPI begonnen werden (z.B. Omeprazol $80 \mathrm{mg}$ i.v.).

Injektionstherapie | Diese Form der endoskopischen Therapie wird am häufigsten angewandt. Dabei führt v.a. der komprimierende Effekt der injizierten Substanzen zur Hämostase.

Die submuköse Injektion von verdünnter Adrenalinlösung (1:10000 oder 1:100 000) ist die häufigste Form der Injektionstherapie.

Diese Methode ist flächendeckend verfügbar, einfach, kostengünstig und effektiv. Es konnten jedoch keine Unterschiede in der Hämostaserate zwischen Natriumchlorid- und Adrenalinlösung beobachtet werden - der durch Adrenalin bewirkte vasokonstriktive Effekt spielt also keine wesentliche Rolle.

Fibrinkleber I Bei der Injektion von Fibrinkleber werden die Komponenten Fibrinogen und Thrombin entweder hintereinander mit einer zwischengeschalteten Natriumchloridspülung oder über ein doppellumiges Nadelsystem simultan appli-

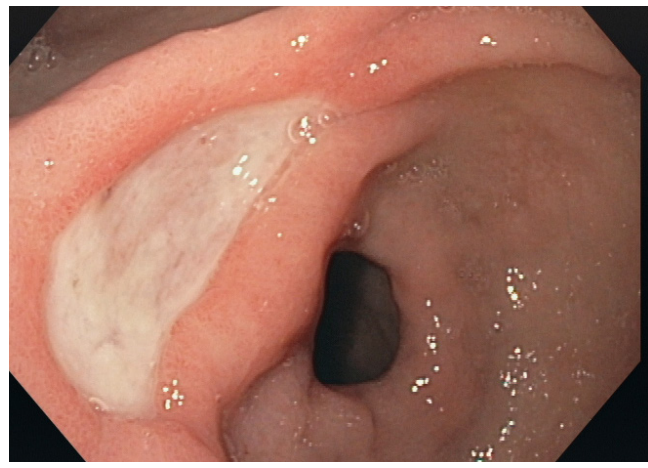

Abb. 6 Forrest-III-Ulkus (fibrinbelegt).

ziert. Dies hat einerseits eine komprimierende und andererseits eine verklebende Wirkung. Es besteht jedoch kein Vorteil gegenüber der Adrenalininjektion. Nachteilig sind zudem

- die schwerere Anwendbarkeit (Produkt muss aufgetaut werden, die Injektion führt häufig zur Okklusion des Nadelsystems),

- die hohen Kosten und

- die Tatsache, dass es sich um ein humanes Plasmaprodukt handelt, das dem Transfusionsgesetz unterliegt und potenziell zu Infektionen führen kann (HIV, HCV, HBV).

Sklerosierung I Weder in der alleinigen noch in der zusätzlichen Gabe zeigen Sklerosierungsverfahren, z.B. mittels Polidocanol oder absolutem Alkohol, einen Vorteil gegenüber der alleinigen Adrenalininjektionen. Auch die Komplikationsrate ist höher, weshalb sie nicht mehr empfohlen werden. Ebenfalls keinen Vorteil gegenüber der Injektionstherapie oder der mechanischen Therapie zeigen thermische Verfahren wie die Argon-Plasma-Koagulation.

Metallclips applizieren | Ein mechanisches Therapieverfahren ist das Applizieren von Metallclips [11], vor allem beim Nachweis von Blutungen aus sichtbaren Gefäßstümpfen (Forrest Ia, Ila). Dabei werden die Clips über ein Applikationssystem

- durch den Arbeitskanal vorgebracht und ausgefahren,

- gespannt auf die Blutungsquelle gedrückt,

- verschlossen und

- vom Applikationssystem gelöst.

Die initalen Hämostaseraten sind vergleichbar mit denen der Injektionstherapie.

Allerdings erzielt die Hämoclipmethode eine signifikant bessere definitive Hämostase, geringere Rezidivblutungswahrscheinlichkeit und eine geringere OP-Rate.

Limitationen der Metallclip-Therapie I Die Bedeutung der Metallclip-Applikation wird aber überschätzt, da häufig die Bedingungen den Einsatz limitieren wie $z$. B. 
- ungünstige intraluminale Raumverhältnisse oder

- ein derber Ulkusgrund, in dem ein Clip keinen Halt findet.

Therapien kombinieren I Die Kombination von Injektionstherapie mit anderen Techniken wie Fibrinkleber oder Metallclips ist der alleinigen Injektion überlegen [12].

Eine Kombination zweier endoskopischer Blutstillungsverfahren gilt daher als Standardtherapie.

Nanopulver | Bei dieser neuen Therapieoption zur Blutstillung appliziert man ein biokompatibles Polysaccharid über einen Sprühkatheter auf die Blutungsfläche. Es nimmt nach dem Aufsprühen in Verbindung mit Feuchtigkeit einen gelartigen Zustand an - so wird die Blutungsquelle abgedeckt. Verschiedene Studien überprüfen die Wertigkeit dieser Methode. Es scheint aber eine sinnvolle Therapieoption z. B. bei schwer einstellbaren Blutungsquellen oder schwer beherrschbaren flächenhaften Sickerblutungen zu sein [13].

Helicobacter bestimmen | Schon während einer Initial-ÖGD sollten Biopsien zur frühzeitigen Helicobacter-Bestimmung entnommen werden: Eine Eradikationstherapie senkt die Wahrscheinlichkeit für Rezidivblutungen $[14,15]$.

Verlauf | Nach initialer Hämostase lässt sich die Inzidenz von Blutungsfrührezidiven reduzieren, indem die PPI-Gabe fortgeführt wird. Dabei ist die Bolusgabe der Dauerinfusion gleichwertig. Anschließend folgt die orale PPI-Therapie als einfache Standarddosis für etwa 6 Monate im Sinn einer Dauerrezidivprophylaxe. Eine Rezidivblutung nach endoskopischer Blutstillung sollte viszeralchirurgisch abgeklärt werden.

\section{Therapie der Varizenblutung}

Intensivbetreuung | Bei Verdacht auf Varizenblutung muss der Patient prinzipiell intensivmedizinisch mit Schutzintubation versorgt werden.

Pharmakologische Therapie | Schon präinterventionell sollen initial Vasopressinanaloga (Terlipressin oder Somatostatin) gegeben und über 3-5 Tage fortgeführt werden. Diese reduzieren über Konstriktion der Splanchnikusgefäße den Pfortaderfluss und somit den Druck im Bereich des Pfortadersystems. Dadurch sinkt auch der Druck im Bereich der ösophagogastralen Varizen. Zusätzlich stabilisiert die Therapie den Kreislauf und fördert die renale Perfusion.
Die pharmakologische Therapie bewirkt bei akuter Varizenblutung eine vergleichbare Hämostaserate wie die alleinige endoskopische Therapie.

Pharmakologisch und endoskopisch | Die Kombinationstherapie bietet hingegen einen Vorteil bei der initialen Blutungskontrolle und der 5-Tage-Hämostase, ohne die Gesamtmortalität oder Nebenwirkungsrate im Vergleich zur alleinigen endoskopischen Therapie zu beeinflussen. Terlipressin kann man alternativ auch kontinuierlich über einen Perfusor verabreichen, um kardiovaskuläre Nebenwirkungen zu minimieren. Außerdem gibt es Hinweise für eine vergleichbar effektive Wirkung bei einer Verkürzung der Therapiedauer auf 24 Stunden $[16,17]$ ( $\bullet$ Tab. 3).

Antibiotika I Die präinterventionelle antibiotische Therapie mittels Chinolon oder 3. Generations-Cephalosporin reduziert die infektionsbedingte Mortalität (spontan bakterielle Peritonitis, Pneumonie, Harnwegsinfekt) und Rezidivblutungsrate - die Therapie dauert 7 Tage [18]. Aufgrund der höheren Resistenzraten für Chinolone empfiehlt sich eine Therapie mit Ceftriaxon.

Protonenpumpenhemmer I Eine PPI-Therapie parallel zur Ligaturtherapie bis zur Eradikation der Varizen

- beschleunigt die Abheilung von Ligaturulzera und

- verringert Rezidivblutungsrate und Mortalität,

- erhöht dabei jedoch nicht das Auftreten von PPI-bedingten Infektkomplikationen.

Laktulose I Die Gabe von Laktulose reduziert das Risiko einer blutungsbedingten Enzephalopathie bei Patienten mit Leberzirrhose und kann initial am Ende der Notfallendoskopie gegeben werden.

Varizenligatur I Bei akuter Ösophagusvarizenblutung ist die endoskopische Varizenligatur (EVL) die Therapie der Wahl [19]. In besonders schweren Fällen kann alternativ Histoacryl (Nbuthyl-cyanoacrylate) injiziert werden, wobei man jedoch das Embolierisiko beachten muss.

\begin{tabular}{|c|c|}
\hline Zeitpunkt & Maßnahme \\
\hline \multirow[t]{3}{*}{ präinterventionell } & Kreislaufstabilisierung, Schutzintubation \\
\hline & Vasopressinanaloga (bis 5 Tage) \\
\hline & Antibiose: Cephalosporin der 3. Generation (7 Tage) \\
\hline \multirow[t]{2}{*}{ endoskopisch } & $\begin{array}{l}\text { Ösophagusvarizen: EVL, alternativ Histoacryl- } \\
\text { Injektion }\end{array}$ \\
\hline & $\begin{array}{l}\text { Magenvarizen: Histoacryl (GOV2, IGV), alternativ } \\
\text { EVL (GOV1) }\end{array}$ \\
\hline $\begin{array}{l}\text { bei Therapieversagen } \\
\text { oder frühem Rezidiv }\end{array}$ & $\begin{array}{l}\text { transjugulärer intrahepatischer portosystemischer } \\
\text { Shunt (TIPS) }\end{array}$ \\
\hline
\end{tabular}

Tab. 3 Verschiedene Möglichkeiten der Blutungsstillung bei Ösophagusvarizen [11, 18-21]. 


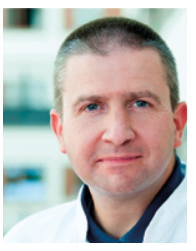

Dr. med. Robert Baumbach ist Oberarzt an der Abteilung für Gastroenterologie und Interventionelle Endoskopie, Asklepios Klinik Barmbek, Hamburg.

r.baumbach@asklepios.com

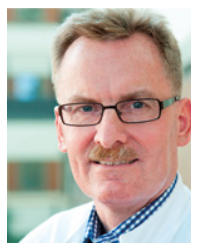

PD Dr. med. Siegbert Faiss ist Chefarzt der Abteilung für Gastroenterologie und Interventionelle Endoskopie, Asklepios Klinik Barmbek, Hamburg.

s.faiss@asklepios.com

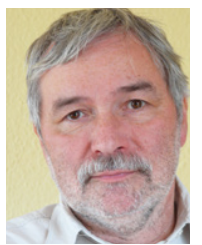

Wolfgang Cordruwisch ist Oberarzt an der Abteilung für Gastroenterologie und Interventionelle Endoskopie, Asklepios Klinik Barmbek, Hamburg. w.cordruwisch@ asklepios.com

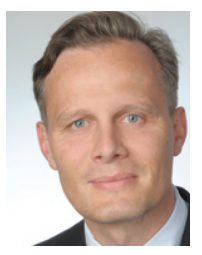

PD Dr. med. Carsten

Schrader

ist niedergelassener Internist in Kiel.

c.schrader@asklepios.com

DOI 10.1055/s-0042-101721

VNR2760512016149753968

Dtsch Med Wochenschr

2016; 141: 561-570

(c) Georg Thieme Verlag KG .

Stuttgart $\cdot$ New York .

ISSN 0012-0472
Beide Verfahren unterscheiden sich nicht in Hämostase, Überleben oder Rezidivblutungsrate. Die Sklerosierungstherapie mit Ethoxysklerol wird nicht mehr empfohlen, da sie im Vergleich zur EVL eine höhere Komplikationsrate hat (Ulzera, Perforation, Mediastinitis) [20].

Injektionstherapie | Bei gastroösophagealen Varizen Typ 2 (GOV2, bis in den Fundus reichend) und isolierten gastralen Varizen (IGV) ist die intravasale Injektion von Histoacryl Therapie der Wahl. Sie ist hinsichtlich Hämostase, Rezidivblutungsrate und Mortalität der EVL überlegen. Bei gastroösophagealen Varizen Typ 1 (GOV1, im Bereich der Kardia) sind EVL und Histoacryl-Injektion gleichwertige Methoden [21].

Vorübergehende Ballontamponade I Kann die Kombination aus Pharmakotherapie und endoskopischer Therapie keine Stabilität über 6 Stunden erzielen oder tritt frühzeitig eine Rezidivblutung auf, kann vorübergehend eine Ballontamponade gelegt werden:

- Sengstaken-Sonde bei Ösophagusvarizen

- Linton-Nachlass-Sonde bei gastrischen Varizen Aufgrund der Gefahr von Druckulzera dürfen diese Sonden höchstens 24 Stunden liegen.

TIPS | Eine Alternative zur Sondeneinlage ist ein voll gecoverter selbstexpandierender Metallstent (Ella-Danis-Stent). Dieser wird ohne Röntgenkontrolle mit einem Positionierungsballon freigesetzt und bis zu 14 Tage belassen [22]. Nach Entfernung der Stents zeigt sich jedoch häufiger ein Blutungsrezidiv. Diese Methode ist daher allenfalls eine Bridging-Maßnahme bis zu einer definitiven Therapie mittels transjugulärem intrahepatischen portosystemischen Shunt (TIPS) [23-25]. Zudem reduziert der frühelektive TIPSEinsatz bei Ösophagusvarizenblutung nach erfolgreicher endoskopischer Blutstillung signifikant die Rezidivblutungen und die Mortalität.

Eine TIPS-Anlage wird daher bei einer Hochrisikoblutung innerhalb von 72 Stunden (ideal < 24 Stunden) nach initialer pharmakologischer und endoskopischer Therapie empfohlen [21].

Operation I Die Notfall-Laparotomie als invasivste Methode steht als Ultima Ratio zur Verfügung, wenn endoskopisch oder radiologisch keine Blutstillung sinnvoll oder möglich ist. Typische Bespiele sind blutende gastrointestinale Stromatumore oder unstillbare bzw. rezidivierend blutende Ulzera der Bulbushinterwand (Blutung aus der A. gastroduodenalis) [26].

\section{Konsequenz für Klinik und Praxis}

- Die Gl-Blutung ist ein häufiger internistischgastroenterologischer Notfall. Die meisten Gl-Blutungen sind im oberen Gl-Trakt lokalisiert.

- Die Prognose hängt v. a. von Blutungsintensität, Alter, Komorbidität und Einnahme von Antikoagulation ab.

- Leitsymptome in Verbindung mit Parameter für die Blutungsintensität geben wichtige Hinweise zur Blutungslokalisation.

- Bevor die Blutung gestillt und ein Rezidiv verhindert wird, ist das dringlichste Ziel die Kreislaufstabilisierung.

- Die Ösophagogastroduodenoskopie ist die Methode der Wahl für die Lokalisierung der Blutungsquelle und interventionellen Therapie.

- Blutungen durch peptische Ulzera werden mit PPI und endoskopisch in erster Linie mit Adrenalininjektion, ggf. kombiniert mit einem weiteren thermischen oder mechanischen Verfahren therapiert.

- Bei nicht beherrschbarer Gl-Blutung ist die Operation die Ultima Ratio.

\section{Literatur}

1 Kurien M, Lobo AJ. Acute upper gastrointestinal bleeding. Clin Med 2015; 15: 481-485

2 Ell C, Hagenmuller F, Schmitt W et al. Multicenter prospective study of the current status of treatment for bleeding ulcer in Germany. Dtsch Med Wochenschr 1995; 120: 3-9

3 Rehman A, Iscimen R, Yilmaz M et al. Prophylactic endotracheal intubation in critically ill patients undergoing endoscopy for upper $\mathrm{Gl}$ hemorrhage. Gastrointest Endosc 2009; 69: e55-e59

4 Bai Y, Guo JF, Li ZS. Meta-analysis: erythromycin before endoscopy for acute upper gastrointestinal bleeding. Aliment Pharmacol Ther 2011; 34: 166-171

5 Szary NM, Gupta R, Choudary A et al. Erythromycin prior to endoscopy in acute upper gastrointestianl bleeding: a meta-analysis. Scand J Gastroenterol 2011; 46: 920-924

6 Pateron D, Vicaut E, Debuc E et al. Erythromycin infusion or gastric lavage for upper gastrointestinal bleeding: a multicenter randomized controlled trial. Ann Emerg Med 2011; 57: 582-589

7 Forrest JA, Finlayson ND, Shearman DJ. Endoscopy in gastrointestinal bleeding. Lancet 1974; 2: 394-397

8 Myburgh JA, Finfer S, Bellomo R et al. Hydroxyethyl starch or saline for fluid resuscitation in intensive care. N Engl J Med 2012; 367: 1901-1911

Interessenkonflikt

Die Autoren geben an, dass kein Interessenkonflikt besteht.

Vollständiges Literaturverzeichnis unter http://dx.doi.org/10.1055/s-0042-101721 
9 Bundesärztekammer. Querschnitts-Leitlinien (BÄK) zur Therapie mit Blutkomponenten und Plasmaderivaten, 4. Aufl., Deutscher Ärzte-Verlag; 2014. Im Internet: http://www.bundesaerztekammer.de/ fileadmin/user_upload/downloads/QLL_ Haemotherapie_2014.pdf

10 Bae S. Incidence and 30-day mortality of peptic ulcer bleeding in Korea. Eur J Gastroenterol Hepatol 2012; 24: 675-682

11 Gevers AM, De GE, Simoens M et al. A randomized trial comparing injection therapy with hemoclip and with injection combined with hemoclip for bleeding ulcers. Gastrointest Endosc 2002; 55 : 466-469

12 Barkun AN, Bardou M, Kuipers EJ et al. International consensus recommendation on the management of patients with nonvariceal upper gastrointestinal bleeding. Ann intern Med 2010; 152: 101-113

13 Sung JJ, Luo D, Wu JC et al. Early clinical experience of the safety and effectiveness of Hemospray in achieving hemostasis in patients with acute peptic ulcer bleeding. Endoscopy 2011; 43: 291-295

14 Lau JY, Sung JJ, Lee KK et al. Effect of intravenous omeprazole on recurrent bleeding after endoscopic treatment of bleeding peptic ulcers. N Engl J Med 2000; 343: 310-316

15 Lau JY, Leung WK, Wu JC et al. Omeprazole before endoscopy in patients with gastrointestinal bleeding. N Engl J Med 2007; 356: 1631-1640

16 Gerbes AL, Huber E, Gulberg V. Terlipressin for hepatorenal syndrome: continuous infusion as an alternative to i. v. bolus administration. Gastroenterology 2009; 137: 1179-1181

17 Gonzalez R, Zamora J, Gomez-Camarero J et al. Meta-analysis: Combination endoscopic and drug therapy to prevent variceal rebleeding in cirrhosis. Ann Intern Med 2008; 149: 109-122

18 Bernard B, Grange JD, Khac EN et al. Antibiotic prophylaxis for the prevention of bacterial infections in cirrhotic patients with gastrointestinal bleeding: a meta-analysis. Hepatology 1999; 29 : 1655-1661
19 Saeed ZA, Stiegmann GV, Ramirez FC et al. Endoscopic variceal ligation is superior to combined ligation and sclerotherapy for esophageal varices: a multicenter prospective randomized trial. Hepatology 1997; 25: 71-74

20 Stiegmann GV, Goff JS, Michaletz-Onody PA et al. Endoscopic sclerotherapy as compared with endoscopic ligation for bleeding esophageal varices. N Engl J Med 1992; 326: 1527-1532

21 de Franchis R, Baveno V Faculty. Revising consensus in portal hypertension: Report of the Baveno $\mathrm{V}$ consensus workshop on methodology of diagnosis and therapy in portal hypertension. J of Hepatology 2010; 53: 762-768

22 Maufa F, Al-Kawas FH. Role of self-expandable metal stents in acute variceal bleeding. Int ] Hepatol 2012; 2012: 418369

23 de Franchis R. Evolving consensus in portal hypertension. Report of the Baveno IV consensus workshop on methodology of diagnosis and therapy in portal hypertension. J Hepatol 2005; 43: 167-176

24 Garcia-Pagan JC, Caca K, Bureau C et al. Early use of TIPS in patients with cirrhosis and variceal bleeding. N Engl J Med 2010; 362: 2370-2379

25 Stanley AJ, Jalan R, Forrest EH et al. Longterm follow up of transjugular intrahepatic portosystemic stent shunt (TIPSS) for the treatment of portal hypertension: results in 130 patients. Gut 1996; 39: 479-485

26 Lau JY, Sung JJ, Lam YH et al. Endoscopic retreatment compared with surgery in patients with recurrent bleeding after initial endoscopic control of bleeding ulcers. N Engl ] Med 1999; 340: 751-756

27 Heining-Kruz S, Finkenzeller T, Schreyer A et al. Transcatheter arterial embolisation in upper gastrointestinal bleeding in a sample of 29 patients in a gastrointestinal referral center in Germany. Z Gastroenterol 2015; 53: 1071-1079

Der Beitrag wurde gemäß folgendem Erratum erratiert:

Im CME-Beitrag „Akute obere gastrointestinale Blutung“ (Dtsch Med Wochenschr 2016; 141: 561-570) wurden folgende Änderungen vorgenommen:

- Im Abschnitt „Alternative bei unbehandelter Blutungsquelle" auf S. 564 wurde folgender Teilsatz hinzugefügt:

„- dies ist allerdings nur in $86 \%$ der Fälle möglich [27].“

- Antwort c, Frage 9 wurde geändert in: „Blutungen aus aortointestinalen Fisteln verlaufen unbehandelt selten tödlich“. 
CME-Teilnahme

- Viel Erfolg bei Ihrer CME-Teilnahme unter http://cme.thieme.de

- Diese Fortbildungseinheit ist 12 Monate online für eine CME-Teilnahme verfügbar.

- Sollten Sie Fragen zur Online-Teilnahme haben, unter http:// cme.thieme.de/hilfe finden Sie eine ausführliche Anleitung.

1. Welches ist die häufigste Ursache einer GI-Blutung?

a Angiodysplasie

b Ösophagusvarizen

c Ulkus

d Gastritis

e Tumor

2. Welche Aussage zur klinischen Einschätzung bei der oberen GI-Blutung ist richtig?

a Patienten können mit einem normalen Hb-Wert verbluten.

b Der Hb-Wert ist ein zuverlässiger Parameter zur Beurteilung der Blutungsintensität.

c Bei einem niedrigen $\mathrm{Hb}$-Wert liegt immer eine aktive Blutung vor.

d Arterielle Hypotonie und Tachykardie sind immer sichere Schockzeichen bei einer GI-Blutung.

e Eine Erythrozytenkonzentratgabe sollte immer erst nach der Bestimmung des Hb-Werts erfolgen.

3. Welche Aussage ist richtig?

a. Die Anamnese spielt bei der Einschätzung der oberen GI-Blutung eine untergeordnete Rolle.

b. Ein Alkoholiker hat meistens eine Ulkusblutung im Magen.

c. Die Ulkusblutungen sistieren meistens spontan.

d. Erst ab $1000 \mathrm{ml}$ Blut kommt es zum Auftreten von Meläna.

e. Meläna ist immer Zeichen einer oberen GIT-Blutung.

4. Welche Aussage zur Stadieneinteilung nach Forrest trifft zu?

a. Die Forrest-III-Blutung bedarf einer akuten endoskopischen Therapie.

b. Forrest-I- und Ila-Ulzera sollten zeitnah endoskopisch behandelt werden.

c. Eine Forrest-IIc-Blutung wird immer mit einem Clip versorgt.

d. Forrest-I- und II-Ulzera bedürfen alle einer endoskopischen Therapie.

e. Eine Forrest-IIa-Blutung hat ein geringes Rezidivrisiko.

5. Welche Aussage zur Angiografie ist richtig?

a. Die Angiografie sollte bei jeder Blutung großzügig durchgeführt werden.

b. Die Angiografie ist immer erst ab einer Blutung von mehr als $5 \mathrm{ml} /$ min detektierbar.

c. Die Angiografie ist nur sinnvoll bei einer Massenblutung.

d. Indirekte Zeichen einer Blutungsquelle können Gefäßmalformationen sein.

e. In der Angiografie kann immer das Gefäß behandelt werden.

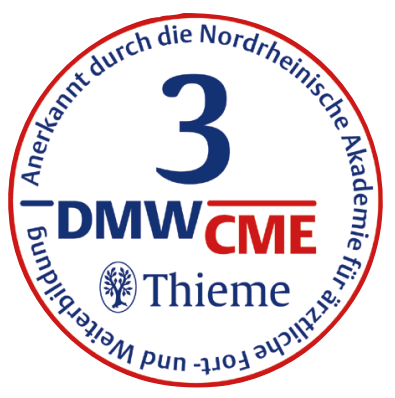

6. Welche Aussage zur Ösophagusvarizenblutung ist falsch?

a. Die Terlipressintherapie ist der endoskopischen Therapie bzgl. der Hämostaserate unterlegen.

b. Eine medikamentöse Therapie sollte vor der endoskopischen beginnen.

c. Wegen der Aspirationsgefahr sollte vor der Endoskopie eine Schutzintubation erwogen werden.

d. Der Einsatz von PPI senkt Rezidivrate und Mortalität.

e. Eine Antibiotikagabe ist indiziert.

7. Welche Aussage zur OP der GI-Blutung ist richtig?

a. Die Operation ist die Methode der Wahl zur Behandlung der oberen GI-Blutung.

b. Eine typische OP-Indikation ist die Ösophagusvarizenblutung.

c. Die operative Behandlung einer GI-Blutung ist häufig.

d. Die OP ist die Ultima Ratio, sofern endoskopisch und radiologisch keine Therapieoptionen bestehen.

e. Häufig finden sich Blutungen im Bereich der kleinen Kurvatur des Magens.

8. Welche Aussage zu Allgemeinmaßnahmen ist falsch?

a. Es sollten zur Kreislaufsicherung mindestens 2 Zugänge gelegt werden.

b. Es sollte ein kontinuierliches Kreislaufmonitoring erfolgen.

c. Auch bei fehlender Luftnot ist eine Sauerstoffgabe sinnvoll.

d. Die Gabe von Erythrozytenkonzentraten sollte ab einem Wert unter $10 \mathrm{~g} / \mathrm{dl}$ erfolgen.

e. Initial ist die Gabe von Elektrolytlösungen sinnvoll.

9. Welche Aussage zu seltene Ursachen einer Blutung ist richtig?

a. Die Blutgerinnung spielt erst bei Magenulzera eine Rolle.

b. Aortointestinale Fisteln finden sich überwiegend im Duodenum.

c. Blutungen aus aortointestinalen Fisteln verlaufen unbehandelt selten tödlich.

d. Die Lokalisation der Blutung eines Ulkus Dieulafoy ist meist das Duodenum.

e. Blutungen aus Angiodysplasien sind häufig.

10. Welche Aussage ist richtig?

a. Bei fehlender Sicht wegen eines Koagels kann die Umlagerung des Patienten die blutende Läsion detektieren.

b. Die Gabe von Prokinetika bringen nie eine verbesserte Sicht.

c. Die Indikation zur Notfall-Gastroskopie sollte nur bei instabilen Patienten erfolgen.

d. Ein Ulkus Forrest I $\mathrm{a}$ und $\mathrm{b}$ bedürfen keiner Therapie.

e. Ein Ulkus Forrest III sollte immer therapiert werden. 


\section{Angaben zur Person}

Name, Vorname, Titel:

Straße, Hausnr:

PLZ, Ort:

Anschrift: $\square$ privat $\square$ dienstlich

Ich bin Mitglied der Ärztekammer (bitte Namen der Kammer eintragen):

Jahr meiner Approbation:

Ich befinde mich in der Weiterbildung zum:

Ich habe eine abgeschlossene Weiterbildung in ...

(bitte Fach eintragen):

Ich bin tätig als: $\square$ Assistenzarzt $\square$ Oberarzt $\square$ Chefarzt $\square$ niedergelassener Arzt $\square$ Sonstiges Ich bin DMW-Abonnent: $\square$ ja $\square$ nein

Falls nein: ich habe den Fragebogen aus / von:

$\square$ Thieme-connect $\square$ Kollegen $\square$ der Klinik $\square$ einer Bibliothek $\square$ Sonstiges

Lernerfolgskontrolle (Eine Antwort pro Frage ankreuzen)
1. $\square$ A $\square$ B $\square$ C $\quad \square$ D $\quad \square$ E
2. $\square$ A $\square$ B $\square$ C $\square$ D $\square \mathrm{E}$
3. $\square \mathrm{A} \quad \square \mathrm{B} \quad \square \mathrm{C} \quad \square \mathrm{D} \quad \square \mathrm{E}$
4. $\square$ A $\square$ B $\square$ C $\square$ D $\square$ E
5. $\square \mathrm{A} \quad \square \mathrm{B} \quad \square \mathrm{C} \quad \square \mathrm{D} \quad \square \mathrm{E}$
6. $\square$ A $\square$ B $\square$ C $\square$ D $\square$ E
7. $\square$ A $\square$ B $\square$ C $\square$ D $\square$ E
8. $\square$ A $\square$ B $\square$ C $\square$ D $\square$ E
9. $\square \mathrm{A} \square \mathrm{B} \quad \square \mathrm{C} \quad \square \mathrm{D} \quad \square \mathrm{E}$
10. $\square \mathrm{A} \quad \square$ B $\square \mathrm{C} \quad \square \mathrm{D} \quad \square \mathrm{E}$

Ich versichere, dass ich die Beantwortung der Fragen selbst und ohne fremde Hilfe durchgeführt habe

Ort, Datum:

Unterschrift:

Bitte in dieses Feld Ihre DMW Abonnement-Nummer eintragen:

\section{Fragen zur Zertifizierung}

1. Das Thema des Beitrages kommt in meiner ärztlichen Tätigkeit $\square$ häufig vor $\square$ selten vor $\square$ regelmäßig vor $\square$ gar nicht vor

2. Bei diesem Thema habe ich $\square$ eine feste Gesamtstrategie $\square$ keine Strategie $\square$ noch offene Einzelprobleme

3. In Bezug auf das Thema des Beitrages

$\square$ fühle ich mich nach dem Studium des Beitrags in meiner Strategie bestätigt

$\square$ habe ich meine Strategie verändert:

$\square$ habe ich erstmals eine einheitliche Strategie erarbeitet

$\square$ habe ich keine einheitliche Strategie ableiten können

4. Wurden aus der Sicht Ihrer täglichen Praxis heraus wichtige Aspekte des Themas

nicht erwähnt: $\square$ ja, welche $\square$ nein

zu knapp abgehandelt? $\square$ ja, welche $\square$ nein

überbewertet? $\square$ ja, welche $\square$ nein

5. Verständlichkeit des Beitrages

$\square$ Der Beitrag ist nur für Spezialisten verständlich

$\square$ Der Beitrag ist auch für Nicht-Spezialisten verständlich

6. Beantwortung der Fragen

$\square$ Die Fragen lassen sich aus dem Studium des Beitrags allein beantworten

$\square$ Die Fragen lassen sich nur unter Zuhilfenahme zusätzlicher Literatur beantworten

7. Die Aussagen des Beitrages benötigen eine ausführlichere Darstellung

$\square$ zusätzlicher Daten

$\square$ von Befunden bildgebender Verfahren

$\square$ die Darstellung ist ausreichend

8. Wieviel Zeit haben Sie für das Lesen des Beitrages und der Bearbeitung des Quiz benötigt?

Zertifizierungsfeld (wird durch die DMW ausgefülltt)

Ihr Ergebnis:

Sie haben

von 10 Fragen richtig beantwortet.

Sie haben $\square$ bestanden und 3 Punkte erworben

$\square$ nicht bestanden $\square$ ungültig, weil:

Stuttgart, den Stempel/Unterschrift 